
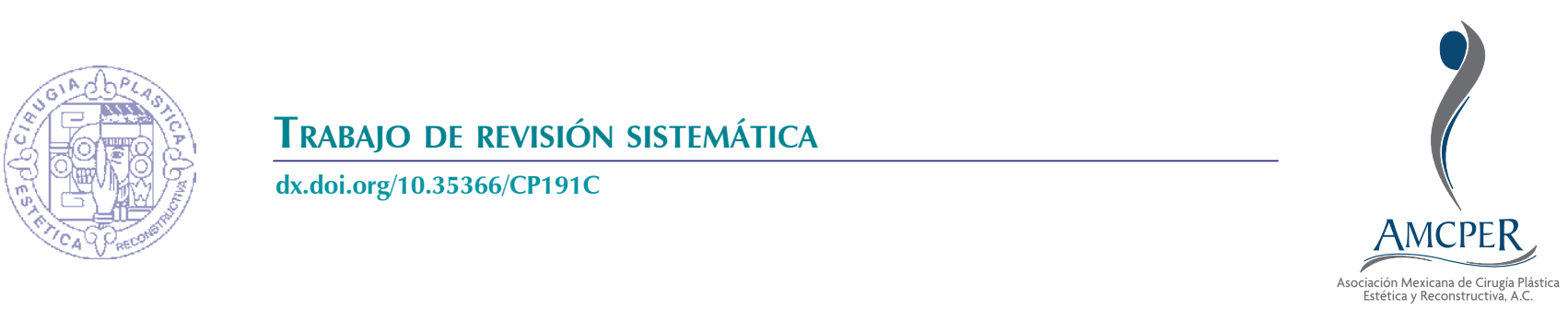

\title{
Estratificación del riesgo de trombosis y profilaxis: ¿cuál es la mejor puntuación para estratificar el riesgo de trombosis en los pacientes de cirugía plástica?, đcuál es la mejor profilaxis? Medicina basada en evidencia
}

\author{
Stratification of the risk of thrombosis and prophylaxis: What is \\ the best score to stratify the risk of thrombosis in patients of plastic \\ surgery? What is the best prophylaxis? Evidence Based Medicine
Dr. Jesús Cuenca-Pardo, ${ }^{*}$ Dr. Guillermo Ramos-Gallardo, ${ }^{\ddagger}$ Dr. Martín Morales Olivera, ${ }^{\ddagger}$ Dr. Javier Bucio-Duarte, ${ }^{\ddagger}$ Dra. Isabel Caravantes-Cortés ${ }^{\ddagger}$

Palabras clave: Trombosis, trombosis venosa profunda, tromboembolia pulmonar, clasificación de riesgo, escalas para calificar riesgo, profilaxis, quimioprofilaxis, cuidados generales.

Keywords: Thrombosis, deep vein thrombosis, pulmonary thromboembolism, risk classification, scores to qualify risk, prophylaxis, chemoprophylaxis, general care.

\footnotetext{
* Asesor del Comité de Seguridad de la Asociación Mexicana de Cirugía Plástica, Estética y Reconstructiva. Coordinador del Comité de Seguridad de la Federación Iberolatinoamericana de Cirugía Plástica (FILACP). Exprofesor adjunto y Titular del Curso de Cirugía Plástica con reconocimiento
}

\section{RESUMEN}

Todos los pacientes que se someten a una cirugía, incluso cirugía facial, tienen el riesgo de que ésta se complique con una trombosis. La trombosis es la principal complicación mortal en aquéllos a los que se realiza abdominoplastia; la incidencia aumenta cuando se combina con liposucción u algún otro tipo de procedimiento. Presentamos una revisión sistemática con tendencia a la Medicina Basada en Evidencia (MBE) para determinar la mejor recomendación que califique el riesgo de trombosis y las mejores recomendaciones profilácticas en los pacientes de cirugía plástica. Tomamos como escenario un caso habitual en cirugía plástica: realizamos un análisis de las clasificaciones actuales, calificamos a la paciente del caso presentado y comparamos los resultados y recomendaciones. Existe gran discrepancia en la estratificación del riesgo de trombosis entre las clasificaciones utilizadas: la calificación más baja fue con la escala de Caprini y la más alta con las de IMPROVE y ACCP. Existe una diferencia notoria en las clasificaciones del riesgo; sin embargo, hay una concordancia en la recomendación del uso de heparina de bajo peso molecular y en los cuidados generales. Las clasificaciones actuales no incluyen factores trombogénicos existentes en los pacientes de cirugía plástica. Mientras no exista una escala efectiva y adaptada a los pacientes de cirugía plástica se deberá utilizar una clasificación existente y agregar los factores trombogénicos de los procedimientos de la especialidad, lo que aumentará la calificación de los pacientes y la indicación de la profilaxis.

\section{ABSTRACT}

All patients who undergo surgery, including facial surgery, are at risk of becoming complicated with thrombosis. Thrombosis is the main fatal complication in patients who undergo tummy tucks; the incidence increases, when combined with liposuction or some other type of procedure. We present a systematic review with an MBE trend, to determine the best recommendation to rate the risk of thrombosis and the best prophylactic recommendations in plastic surgery patients. We took a scenario, a common case in plastic surgery, and analyzed the current classifications, rated the patient of the case presented, and compared the results and recommendations. There is a great discrepancy in the stratification of risk of thrombosis among the classifications used; the lowest grade was with the score of Caprini and the highest with IMPROVE and ACCP. There is a marked difference in risk classifications; however, there is an agreement in the recommendation of the use of low molecular weight heparin and in the general care. Current classifications do not include existing thrombogenic factors in plastic surgery patients. As long as there is no effective score adapted to plastic surgery patients, an existing classification should be used and the thrombogenic factors of the specialty procedures must be added, which will increase the scores of the patients and the indication of prophylaxis. 
universitario en

el Hospital de

Traumatología «Dr.

Victorio de la Fuente

Narváez» (Magdalena de

las Salinas) del IMSS.

‡ Integrante del Comité

de Seguridad de la

Asociación Mexicana de

Cirugía Plástica, Estética

y Reconstructiva.

Los autores de este

artículo no tienen

conflicto de intereses

que declarar.

Recibido:

24 enero 2019

Aceptado para publicar:

04 marzo 2019

\section{ESCENARIO}

$\mathrm{M}$ ujer de 48 años con peso de $84 \mathrm{~kg}$, estatura de 1.60 m, IMC 33.6; G-III, P-I, C-II. Ha tenido síntomas de menopausia desde hace tres años, tratada con estrógenos y fitoestrógenos y otros complementos no especificados. Hace tres días viajó en avión en clase turista con un tiempo de vuelo de cuatro horas; ha tenido moderada elevación de la presión arterial y edema de piernas; niega ser hipertensa. Fue valorada por el cardiólogo, quien señaló hipertensión reactiva. La paciente fue programada para abdominoplastia, liposucción de costados, espalda y región lumbar, así como transferencia de grasa a los glúteos y colocación de implantes mamarios.

¿Cómo determino el riesgo de trombosis?, ¿qué escala debo utilizar?, ¿qué cuidados profilácticos se deben implementar para evitar la trombosis?, ¿se debe dar quimio-profilaxis?, ¿qué medicamentos se deben emplear?, ¿cuándo debo aplicarlos y por cuánto tiempo?

\section{METODOLOGÍA}

Realizamos una revisión sistemática en español e inglés en los sitios de información: PubMed, Embase, Cochrane, Medline, Fisterra, Medigraphic y Google académico. Las palabras clave que utilizamos fueron: thrombosis, thromboembolism, assessment, risk factor, prevention y score para determinar y calificar el riesgo de trombosis, asignando un puntaje y escala de trombosis, comparándolos con las escalas más utilizadas para dar recomendaciones. Asimismo, revisamos publicaciones con nivel de evidencia I, II y III.

\section{INTRODUCCIÓN}

La trombosis o enfermedad trombótica venosa es una de las principales complicaciones mortales en pacientes internados y en pacientes operados. Tiene dos presentaciones: trombosis venosa profunda (TVP) y tromboembolia pulmonar (TEP). Su presentación asintomática tiene una alta incidencia difícil de diagnosticar; en su forma sintomática se ha calculado una incidencia que va desde un caso en 10,000 adultos jóvenes a un caso en 100 adultos ma- yores y la trombosis mortal tiene una incidencia de $0.8 \%$. Los hallazgos en diferentes estudios realizados en autopsias demuestran TVP y TEP en individuos en los que no se había sospechado la enfermedad. ${ }^{1,2}$

La trombosis es la principal complicación mortal en pacientes a los que se realiza abdominoplastia y la incidencia aumenta cuando se combina con liposucción o algún otro tipo de procedimiento. ${ }^{3}$ De manera que la liposucción tumescente del abdomen y de las extremidades inferiores asociado a una prolongada inmovilización puede bloquear el flujo venoso, liberar factores protrombóticos y contribuir a la trombogénesis. ${ }^{2,4-16}$ A pesar de la alta frecuencia de trombosis, el grupo que menos aplica cuidados profilácticos es el de los cirujanos plásticos y quienes los aplican con frecuencia lo hacen en forma insuficiente. ${ }^{2,14-20}$

\section{¿Cómo determinar el riesgo de trombosis?, ¿qué escala debo utilizar?}

Existen varios instrumentos, puntajes o escalas para calificar el riesgo de trombosis, las más utilizadas son la de Caprini/Pannucci, ACCP (CHEST) IMPROVE, Padua y Davison.

\section{Clasificación de riesgo de trombosis. Escala de Caprini}

La escala de Caprini es la más utilizada para la estratificación del riesgo de enfermedad trombótica, se emplea en pacientes quirúrgicos y no quirúrgicos y ha contribuido a establecer cuidados profilácticos y disminuir la incidencia de trombosis venosa profunda y tromboembolia pulmonar. ${ }^{21,22}$

Pannucci y colaboradores realizaron trabajos con la finalidad de validar el instrumento y aplicarlo a pacientes de cirugía plástica. La escala está compuesta por 40 variables repartidas en cinco grupos; cada grupo otorga una puntuación y la calificación final es la suma de todos los factores positivos. Los factores más importantes para esta calificación son la edad y el tiempo de cirugía. Pannucci y su equipo encontraron una incidencia de $0.61 \%$ de tromboembolismo venoso (VTE) en el grupo de $3-4$ puntos, $1.27 \%$ en el grupo de $5-6 ; 2.69 \%$ en el grupo de $7-8$ y $11.32 \%$ en el grupo de 
más de ocho puntos. Los pacientes con una calificación mayor de ocho puntos tenían hasta 20.9 veces más posibilidad de desarrollar VTE, en comparación con aquéllos que tenían una calificación de 3-4 puntos; a mayor puntuación mayor riesgo de trombosis. La ASAPS y la ASPS adoptaron la escala de Caprini modificada por Pannucci y formularon recomendaciones profilácticas (Tabla 1). ${ }^{21-24}$

\section{Consideraciones a la escala de Caprini/ Pannucci relacionada con las pacientes de cirugía plástica a las que se realiza procedimientos estéticos}

1. Incluye muchos factores que en condiciones habituales no están presentes en las pacientes a quienes se realiza un procedimiento estético electivo.

2. El grupo de cuatro puntos no contiene ningún factor.
3. La escala de Caprini/Pannucci no incluye muchos factores. Caprini y colaboradores en la versión 2013 indican que hay que agregar un punto por cada uno de los siguientes factores: obesidad mórbida $<$ de 40, tabaquismo, diabéticos que requieren insulina, quimioterapia, transfusión sanguínea, SIDA $(\mathrm{VIH})$, cirugías de más de dos horas (Tabla 2). ${ }^{22,25}$

4. No incluye factores trombogénicos presentes en las pacientes de cirugía plástica como viajes en avión o autobús previos a la cirugía, realización de abdominoplastia y plastia de la pared abdominal, liposucción e infiltración de grasa, cirugías combinadas o múltiples en el mismo tiempo quirúrgico, grado de movilidad y participación, el uso de medicamentos que aumentan la coagulación como el ácido tranexámico o el etamsilato (utilizados por varios cirujanos para evitar el sangrado) (Tabla 2). ${ }^{15,18,21,26}$

Tabla 1: Escala de Caprini-Pannucci. No hay factores incluidos en el grupo de cuatro puntos.

\begin{tabular}{|c|c|c|c|}
\hline 1 punto & 2 puntos & 3 puntos & 5 puntos \\
\hline Edad de 41-60 años & Edad 60-74 años & Edad mayor 75 años & \multirow{3}{*}{$\begin{array}{l}\text { Fractura de cadera o } \\
\text { miembro pélvico } \\
\text { Reemplazo de cadera o } \\
\text { rodilla } \\
\text { Evento cerebral vascular }\end{array}$} \\
\hline Cirugía menor & $\begin{array}{l}\text { Cirugía mayor (45 } \\
\text { minutos) }\end{array}$ & $\begin{array}{l}\text { Antecedentes de TVP } \\
\text { o TEP }\end{array}$ & \\
\hline $\begin{array}{l}\text { Cirugía mayor último } \\
\text { mes }\end{array}$ & $\begin{array}{l}\text { Malignidad presente o } \\
\text { previa }\end{array}$ & $\begin{array}{l}\text { Historia familiar trom- } \\
\text { bosis }\end{array}$ & \\
\hline Venas varicosas & Cirugía artroscópica & Factor V de Leiden & Mieloma múltiple \\
\hline $\begin{array}{l}\text { Enfermedad inflamatoria } \\
\text { intestinal }\end{array}$ & $\begin{array}{l}\text { Cirugía laparoscópica } \\
\text { (45 minutos) }\end{array}$ & $\begin{array}{l}\text { Presencia de protrombina } \\
\text { 20210A }\end{array}$ & $\begin{array}{l}\text { Lesión de la médula } \\
\text { espinal (parálisis) }\end{array}$ \\
\hline Edema de piernas & - & Homocisteína elevada & - \\
\hline IMC mayor 25 & $\begin{array}{l}\text { Yeso inmovilizador } \\
\text { último mes }\end{array}$ & Anticoagulante de lupus & - \\
\hline $\begin{array}{l}\text { Infarto agudo de } \\
\text { miocardio }\end{array}$ & $\begin{array}{l}\text { Presencia acceso venoso } \\
\text { central }\end{array}$ & $\begin{array}{l}\text { Anticuerpos anticardio- } \\
\text { lipina }\end{array}$ & $\begin{array}{l}\text { SÓLO PARA MUJERES } \\
1 \text { PUNTO }\end{array}$ \\
\hline $\begin{array}{l}\text { Insuficiencia cardiaca } \\
\text { congestiva }\end{array}$ & - & $\begin{array}{l}\text { Trombocitopenia induci- } \\
\text { da por heparina }\end{array}$ & $\begin{array}{l}\text { Anticonceptivos o } \\
\text { terapia hormonal de } \\
\text { reemplazo }\end{array}$ \\
\hline Sepsis último mes & - & $\begin{array}{l}\text { Otra trombofilia congéni- } \\
\text { ta o adquirida }\end{array}$ & $\begin{array}{l}\text { Embarazo o parto último } \\
\text { mes }\end{array}$ \\
\hline Enfermedad pulmonar & - & - & $\begin{array}{l}\text { Abortos espontáneos } \\
\text { recurrentes }\end{array}$ \\
\hline Reposo actual en cama & $\begin{array}{l}\text { Confinamiento en cama } \\
\text { (72 horas) }\end{array}$ & - & - \\
\hline
\end{tabular}


5. Las pacientes menores de 35 años con antecedentes deben ser consideradas de alto riesgo de trombosis, aunque la calificación de Caprini sea baja: infiltración de grasa en los glúteos, venas varicosas, obesidad, colagenopatías, abdominoplastia en el mismo tiempo quirúrgico de una cesárea o parto vaginal, procedimientos múltiples, viajes en avión e ingesta de hormonales. El riesgo aumenta cuando se tiene más de dos de estos factores (Tabla 2). ${ }^{27}$

6. Aunque incluye el índice de masa corporal no lo estratifica, no considera que a mayor IMC mayor riesgo y por lo tanto, deberá ser calificado con una mayor puntuación.

7. El tiempo de duración de la cirugía sólo lo califican en los dos primeros grupos, un punto para cirugía menor y dos puntos para cirugía mayor de 45 minutos. Caprini y colegas $^{22,25}$ aumentan un punto extra a cirugías que duren más de dos horas. El tiempo tiene que estratificarse y ocupar los otros grupos: a mayor tiempo mayor riesgo de trombosis.
Estratificación de riesgo de trombosis y recomendaciones profilácticas de acuerdo a la escala de Caprini-Pannucci nivel de evidencia 2-4; grado de recomendación

$$
\text { B, C y D } \mathbf{D}^{28-40}
$$

En todos los pacientes de cirugía plástica que se hospitalicen o que sean manejados como ambulatorios se deberá realizar una estratificación del riesgo de trombosis, la escala de Caprini/ Pannucci puede ser de utilidad para este fin, las recomendaciones preventivas están basadas en la puntuación del riesgo:

Pacientes con tres puntos. Se debe considerar el uso de heparina de bajo peso molecular en el tiempo que no deambule la paciente.

Pacientes de tres a seis puntos. Heparina de bajo peso molecular o heparina no fraccionada. El uso de quimioprofilaxis postoperatoria durante una semana resulta eficaz en la prevención de trombosis y no aumenta significativamente el riesgo de sangrado.

Pacientes de cirugía electiva con más de siete puntos. Para reducir el riesgo de trombosis se recomienda, previo a la cirugía,

Tabla 2: Lista de factores que no están incluidos en la escala de Caprini-Pannucci.

\begin{tabular}{|c|c|c|}
\hline $\begin{array}{l}\text { A. Factores que deben ser } \\
\text { incluidos en el puntaje según } \\
\text { Caprini y cols.: dar } 1 \text { punto extra } \\
\text { por cada uno }\end{array}$ & $\begin{array}{l}\text { B. Factores trombogénicos } \\
\text { presentes en las pacientes de } \\
\text { la especialidad. Aún no se han } \\
\text { ponderado. Se debe dar por lo } \\
\text { menos } 1 \text { punto cada factor }\end{array}$ & $\begin{array}{l}\text { C. Pacientes menores de } 35 \text { años } \\
\text { con los siguientes antecedentes. Se } \\
\text { debe dar una mayor calificación. } \\
\text { Aún no están ponderadas, pero } \\
\text { debe ser mayor a un punto }\end{array}$ \\
\hline Obesidad mórbida IMC $>40$ & $\begin{array}{l}\text { Viajes en avión o autobús previos a } \\
\text { la cirugía }\end{array}$ & Infiltración de grasa en los glúteos \\
\hline Tabaquismo & Abdominoplastia & Venas varicosas \\
\hline Diabéticos que requieren insulina & Plastia de la pared abdominal & Obesidad \\
\hline Quimioterapia & Liposucción & Colagenopatías \\
\hline Transfusiones sanguíneas & Infiltración de grasa & $\begin{array}{l}\text { Abdominoplastia en el mismo } \\
\text { tiempo quirúrgico de una cesárea } \\
\text { o parto vaginal }\end{array}$ \\
\hline $\mathrm{SIDA}(\mathrm{VIH})$ & Cirugías combinadas o múltiples & Procedimientos múltiples \\
\hline Tiempo de cirugía mayor a dos horas & Grado de movilidad y participación & $\begin{array}{l}\text { Viajes en avión } \\
\text { Ingesta de hormonales }\end{array}$ \\
\hline
\end{tabular}

A: Factores incluidos en la versión 2013 de Caprini. B: Factores trombogénicos en las pacientes sometidas a un procedimiento estético. C: Factores de riesgo en pacientes menores de 35 años. 
disminuir el peso corporal e interrumpir la terapia de reemplazo hormonal. Durante la cirugía reducir el tiempo de duración y el número de procedimientos en el postoperatorio y la movilización postoperatoria temprana. En todos los pacientes se debe utilizar heparina de bajo peso molecular o heparina fraccionada o fondaparinux; la quimioprofilaxis deberá ser de cuatro a seis semanas. ${ }^{30-42}$

\section{Clasificación de riesgo de ACCP (CHEST)}

Para la clasificación del riesgo de trombosis y profilaxis un equipo de trabajo de la American College of Chest Physicians (ACCP), también conocido como CHEST, basado en los criterios del grupo GRADE para determinar la fuerza de las recomendaciones y utilizando como herramienta metodológica los metaanálisis y consensos con expertos, elaboró guías clínicas de alta calidad basadas en la simplicidad, transparencia, metodología explícita y consistencia. Las recomendaciones emitidas las elaboraron con base en el riesgo, con una presentación más resumida y sin menoscabo de la información clave. Las recomendaciones realizadas por los expertos ayudan al juicio clínico basado en sus preferencias y necesidades. La mayoría de los grupos de referencia han aceptado este sistema de clasificación de recomendaciones. El enfoque sistemático ayuda a evitar los sesgos y las interpretaciones equivocadas..$^{43}$ Los resultados actualizados los ha publicado en su octava y novena edición (AT8, AT9: Antithrombotic Therapy and prevention of thrombosis 8th and 9th edition). ${ }^{38-42,44}$ Algunos han realizado adaptaciones a las propuestas por ACCP. ${ }^{2,18,42}$

Asignación del riesgo de trombosis y recomendaciones profilácticas. Nivel de evidencia 1-2; grado de recomendación: 1A, 2A (AT8, AT9) $)^{37-46}$

\section{Riesgo leve}

- Cirugía menor en pacientes con edad menor de 40 años sin factores clínicos de riesgo.

- Riesgo de TVP distal de $2 \%$, proximal $0.4 \%$, TEP $0.2 \%$, TEP mortal $0.002 \%$.

- Recomendación: posición adecuada, ángulo de la rodilla de 10 a 15 grados, pies ligeramente elevados, deambulación temprana.

\section{Riesgo moderado}

- Cirugía menor en pacientes con edad de 40 a 60 años sin otros factores adicionados. Cirugía mayor en pacientes menores de 40 años, sin factores de riesgo.

- Riesgo de TVP distal de $10-20 \%$, proximal $2-4 \%$, TEP $1-2 \%$, TEP mortal $0.1-0.4 \%$.

- Recomendaciones: posición adecuada, ángulo de la rodilla de 10 a 15 grados, pies ligeramente elevados, deambulación temprana, compresión neumática intermitente, medias elásticas.

\section{Riesgo alto}

- Cirugía menor en pacientes mayores de 60 años con factores de riesgo. Cirugía mayor en pacientes mayores de 40 años o con otros factores de riesgo.

- Riesgo de TVP distal de 20-40\%, proximal $4-8 \%$, TEP 2-4\%, TEP mortal 0.4-1\%.

- Recomendaciones: posición adecuada, ángulo de la rodilla de 10 a 15 grados, pies ligeramente elevados, deambulación temprana, compresión neumática intermitente, medias elásticas, considerar la heparina de bajo peso molecular.

\section{Riesgo muy alto}

- Cirugía mayor en pacientes mayores de 40 años con varios factores de riesgo.

- Riesgo del 10-20\%, TVP distal de 40-80\%, proximal $10-20 \%$, TEP $10 \%$, TEP mortal $1-5 \%$.

- Recomendaciones: posición adecuada, ángulo de la rodilla de 10 a 15 grados, pies ligeramente elevados, deambulación temprana, compresión neumática intermitente, medias elásticas, heparina de bajo peso molecular, considerar la warfarina.

\section{Consideraciones relevantes de la octava y novena edición del ACCP (AT8, AT9) $)^{37-42,44}$}

- Se consideró que la identificación de la trombosis asintomática era un parámetro 
muy importante para valorar el resultado de la profilaxis. ${ }^{44}$ Por primera vez se realizó un estudio sobre el diagnóstico de la TVP, aún en la etapa de sospecha. La búsqueda intencional de trombosis en todos los pacientes operados aumentará la detección y tratamiento oportuno, habrá mayores fundamentos para entender la enfermedad y su profilaxis. El uso del ultrasonido Doppler es un recurso no caro para este fin.

- Se sugiere adaptar la escala de estratificación de riesgo a la especialidad u hospital donde se trabaje. Es importante clasificar los procedimientos de cirugía plástica de acuerdo al riesgo y establecer una estrategia formal en el estudio y profilaxis de la trombosis (8AT).

- Los métodos mecánicos deben usarse, preferentemente, en pacientes con riesgo de sangrado o como coadyuvante con los anticoagulantes. (8AT, 9AT).

- En la aceptación de las recomendaciones se tiene que valorar la otra parte del modelo de la coagulación, el riesgo de sangrado.

- La aplicación de ácido acetilsalicílico (ASA) tiene poca utilidad, combinada con otros anticoagulantes tiene mayor riesgo de sangrado, incluyendo del tubo digestivo (8AT y 9AT).

- En pacientes con cirugía mayor, utilizar heparina de bajo peso molecular o heparina no fraccionada o fondaparinux, asociada a métodos mecánicos (8AT, 9AT).

- Se debe tener especial cuidado en el manejo de la quimioprofilaxis en aquéllos con bloqueo regional (8AT).

- El tiempo de duración de la quimioprofilaxis en los pacientes de bajo riesgo son 10 días. En pacientes de alto riesgo y poca movilidad debe extenderse hasta 35 días; en los primeros días se puede utilizar heparina y después antagonistas de la vitamina K. No indican el tiempo en el que se debe iniciar la quimioprofilaxis.

- Todos los pacientes deberán tener una estratificación del riesgo de trombosis; las escalas existen, pero no están bien fundamentadas.

- No hay una definición conceptual de la denominada cirugía mayor y cirugía menor. ${ }^{47}$ Los procedimientos de cirugía plástica deberán ser clasificados de acuerdo a las evidencias y a un consenso de expertos; se deberá considerar que la realización de varios procedimientos en el mismo tiempo quirúrgico aumenta el riesgo y requiere mayores cuidados profilácticos.

¿Cómo podemos determinar si el procedimiento que realizamos debe ser considerado como cirugía mayor?

No existe una uniformidad en el término. Algunos lo han utilizado para cirugía abdominal o torácica, otros lo relacionan con la duración de la cirugía, lo traumático, con la pérdida sanguínea o con la complejidad del procedimiento. Caprini/Pannucci consideran como cirugía mayor a los procedimientos que duran más de 45 minutos. ${ }^{21-23}$ De acuerdo con los criterios de Caprini, la mayoría de los procedimientos de cirugía plástica deben ser considerados como cirugía mayor. ${ }^{44-47}$

1. Cirugía de riesgo menor: procedimientos no invasivos con mínima pérdida de sangre $(<$ $250 \mathrm{~mL}$ ). Riesgo mínimo para el paciente, independiente de la anestesia: cirugías de piel y tejido celular subcutáneo.

2. Riesgo mediano o moderado: procedimientos limitados en cuanto a su naturaleza invasiva, pérdida sanguínea mínima menor a $500 \mathrm{~mL}$. Bajo riesgo propio del paciente (independiente de la anestesia): ingreso limitado a tórax, abdomen, cuello o extremidades. Objetivos diagnósticos o terapia menor, sin resección o alteración importante de órganos. Ejemplos: laparoscopia exploratoria o lisis de adherencias, procedimientos superficiales extensos como cirugía plástica de la cara o extremidades.

3. Riesgo alto o mayor: procedimiento invasivo con pérdida sanguínea menor a $1,500 \mathrm{~mL}$, paciente con riesgo moderado (independiente de la anestesia). Ejemplos: apertura del abdomen como colecistectomía, cirugía de resección o reconstructiva del aparato digestivo; cirugía ortopédica de cadera, hombro o rodilla, como cirugía de reemplazo articular; procedimientos de cirugía plástica como abdominoplastia, liposucción extensa, procedimientos combinados. ${ }^{15,20}$ 
4. Riesgo muy alto o severo: procedimientos invasivos con pérdida sanguínea mayor a 1,500 mL. Ejemplo: procedimientos cardiotorácicos, cirugía de corazón abierto, resección pulmonar; cirugía intracraneal, resección de tumores de cabeza y cuello; cirugía vascular, esquelética o neurológica como aneurisma de aorta, reparación de escoliosis; cirugía craneofacial, cirugía del gran quemado, reconstrucción de mamas/ abdomen. ${ }^{15,20}$

Clasificación de riesgo de trombosis. Escala de Caprini modificada por Davison. Nivel de evidencia 3, 4; recomendación C-D ${ }^{43,44}$

Davison menciona que todas las pacientes de cirugía plástica están expuestas a trombosis, incluso aquéllas a quienes se les realiza lifting facial. Hace referencia a un estudio en el año 2001 en EUA, en el que encontraron 485 casos de TVP y 199 de TEP en pacientes a las que se les realizó estiramiento facial. El $83.7 \%$ fueron operadas con anestesia general y $16.3 \%$ con sedación y anestesia local, por lo que a todas las pacientes se les debió estratificar el riesgo y aplicar cuidados preventivos de trombosis.

Davison utiliza como base de su clasificación la escala de Caprini, presenta un sistema de puntuación fácil de usar para asignar un grupo de riesgo. Es un modelo de evaluación del riesgo con modificaciones específicas perteneciente a la cirugía plástica. El modelo de evaluación del riesgo se divide en tres pasos:

El primer paso se refiere a la «exposición» a factores de riesgo asociados con el contexto clínico. El segundo paso consiste en evaluar los factores de riesgo «predisponentes» asociados al paciente y el tercer paso, las puntuaciones de los pasos uno y dos se suman para obtener una puntuación global para asignar un grupo de riesgo y recomendaciones profilácticas específicas (Tabla 3).

Consideraciones a la escala de Davison. El autor trató de simplificar la escala y presentarla en una forma más organizada. No utilizó una metodología para validar las modificaciones, aunque menciona que está dirigido a calificar el riesgo de trombosis en pacientes de cirugía plástica. Al igual que Caprini no incluyó muchos factores trombogénicos que están presentes en los pacientes de la especialidad, por lo que esta clasificación resulta de poca utilidad.

Clasificación del riesgo de trombosis de acuerdo a IMPROVE (International Medical Prevention Registry on Venous Thromboembolism). Nivel de evidencia 1-3, grado de recomendación $\mathrm{A}, \mathrm{B}, \mathrm{C}^{48}$

IMPROVE es un organismo internacional que incluye 52 hospitales y 12 países, utiliza un programa electrónico de fácil uso y de libre acceso. Además de estratificar el riesgo de trombosis, también estratifica el riesgo de sangrado; utiliza pocos factores, los considerados como más importantes. Para la calificación de trombosis se busca: trombosis venosa profunda previa (VTE), trombofilia, parálisis de miembros inferiores, cáncer, problemas prostáticos, inmovilización igual o mayor de siete días, edad mayor de 60 años. Para el sangrado: úlcera gastroduodenal, hemorragia tres meses previos a la admisión, descenso de plaquetas, falla hepática, catéter venoso central, enfermedad reumática y cáncer actual. Las pruebas de diagnóstico que recomienda para detectar trombosis venosa son el diagnóstico clínico, la prueba de captación de fibrinógeno (exploración de la pierna), la pletismografía de impedancia (GPI), la ecografía Doppler, la ecografía dúplex y la venografía.

Las recomendaciones profilácticas las realizan de acuerdo al grado de riesgo de los pacientes:

Pacientes de bajo riesgo. Pacientes menores de 40 años que se someten a operaciones menores, anestesia general que dura menos de 30 minutos. Para la protección de estos pacientes, se recomienda la ambulación temprana.

Pacientes de riesgo moderado. Pacientes mayores de 40 años que se someten a operaciones importantes que requieren anestesia que dura más de 30 minutos, pero que no tienen factores de riesgo adicionales. Se puede proporcionar una protección adecuada para estos pacientes con medias de compresión graduada, dosis bajas de heparina no fraccionada o heparina de bajo peso molecular y compresión neumática intermitente.

Pacientes de alto riesgo. Pacientes mayores de 40 años con factores de riesgo y/o cirugías de 


\section{Tabla 3: Puntaje de Davison. En lugar de puntos utiliza factores.}

Paso 1. Determinar la exposición a factores de riesgo

\begin{tabular}{llll}
\hline 1 factor & 2 factores & 3 factores & 5 factores \\
\hline Cirugía menor & Cirugía mayor & $\begin{array}{l}\text { Previo infarto del } \\
\text { miocardio } \\
\text { Falla cardiaca congestiva }\end{array}$ & $\begin{array}{l}\text { Fractura de cadera, } \\
\text { pelvis o pierna } \\
\text { Ataque cardiaco }\end{array}$ \\
$\begin{array}{l}\text { Inmovilización por mol- } \\
\text { des de yeso } \\
\text { Paciente confinado en } \\
\text { cama por }>\text { 72 horas } \\
\text { Acceso venoso central }\end{array}$ & Sepsis severa & Trauma múltiple \\
& Colgajo libre & $\begin{array}{l}\text { Lesión aguda de la mé- } \\
\text { dula espinal }\end{array}$ \\
\hline
\end{tabular}

Paso 2. Determinar los factores de riesgo predisponentes

\begin{tabular}{|c|c|c|}
\hline Entorno clínico & Inherentes & Adquiridos \\
\hline Edad de 40-60 años: 1 factor & $\begin{array}{l}\text { Cualquier desorden genético de } \\
\text { hipercoagulabilidad: } 3 \text { factores }\end{array}$ & Lupus anticoagulante: 3 factores \\
\hline Edad $>60$ años: 2 factores & & $\begin{array}{l}\text { Anticuerpos antifosfolípidos: } 3 \\
\text { factores }\end{array}$ \\
\hline Historia de trombosis: 3 factores & & $\begin{array}{l}\text { Desordenes mieloproliferativos: } \\
3 \text { factores }\end{array}$ \\
\hline $\begin{array}{l}\text { Embarazo o postparto > } 1 \text { mes: } \\
1 \text { factor }\end{array}$ & & $\begin{array}{l}\text { Inducción de trombocitopenia por } \\
\text { heparina: } 3 \text { factores }\end{array}$ \\
\hline Enfermedad maligna: 2 factores & & Hiperviscosidad: 3 factores \\
\hline Obesidad > 20\% IBM: 1 factor & & Homocisteinemia: 3 factores \\
\hline $\begin{array}{l}\text { Anticonceptivos orales o terapia } \\
\text { de reemplazo hormonal: } 1 \text { factor }\end{array}$ & & \\
\hline
\end{tabular}

Paso 3. Recomendaciones profilácticas de acuerdo a la clasificación del riesgo:

\begin{tabular}{ccl}
\hline Grado de riesgo & Número de factores & Recomendaciones \\
\hline Bajo & 1 & $\begin{array}{l}\text { Deambulación temprana } \\
\text { Presión neumática intermitente y medias elásticas hasta la } \\
\text { deambulación total }\end{array}$ \\
Alto & 2 & $\begin{array}{l}\text { Presión neumática intermitente y medias elásticas hasta la } \\
\text { deambulación total } \\
\text { Muy alto }\end{array}$ \\
& $3-4$ & $\begin{array}{l}\text { Presión neumática intermitente y medias elásticas hasta la } \\
\text { deambulación total } \\
\text { Heparina de bajo peso molecular iniciar a las } 12 \text { horas postoperatorio }\end{array}$ \\
\end{tabular}

alto riesgo como cirugía abdominal, cirugía ginecológica, operaciones urológicas, especialmente la prostatectomía transvesical. La protección adecuada es el uso heparina no fraccionada de dosis baja, heparina de bajo peso molecular y compresión neumática intermitente. La adición de medias de compresión graduadas a estas medidas puede proporcionar protección adicional. 
Cirugía de muy alto riesgo. Cirugía ortopédica de cadera o rodilla y la reparación de fracturas de cadera. Los pacientes seniles y los factores adicionales aumentan el riesgo de trombosis. Otro tipo de cirugía de muy alto riesgo son las operaciones para extirpar tumores malignos en la región torácica. En general, la protección adecuada para pacientes de muy alto riesgo puede proporcionarse utilizando heparina de bajo peso molecular o warfarina. La adición de medias de compresión neumática intermitente y de compresión graduada a estos agentes puede brindar protección adicional.

\section{Consideraciones a la clasificación de riesgo de trombosis de IMPROVE}

- Utiliza una escala electrónica. Al anotar los datos del paciente emite una calificación de riesgo.

- Recibe información de muchos centros hospitalarios de todo el mundo.

- Es un equipo que trabaja con una metodología clínica y permanece actualizado.

- Estudia la trombosis y también el riesgo de sangrado, trata de mantener un equilibrio entre estas dos entidades.

- No están incluidos los pacientes y procedimientos de cirugía plástica. Para beneficiarse de esta estratificación de riesgo de trombosis habrá que agregar los factores trombogénicos relacionados a los procedimientos de cirugía plástica.

Clasificación del riesgo de trombosis de acuerdo a la escala de Padua. Nivel de evidencia 3-4; grado de recomendación C- ${ }^{49}$

Es un sistema electrónico disponible que utiliza 11 factores y calcula el riesgo en forma ordinal como riesgo leve, riesgo moderado o riesgo alto de trombosis.

\section{Clasificación de riesgo de Anderson. Nivel de evidencia 3-4; Recomendación C-D ${ }^{39,41,42,43}$}

Los autores estudiaron los factores relacionados a la incidencia de trombosis, encontraron que no todos los factores tienen el mismo valor. Consideraron que el riesgo está sujeto principalmente a dos factores: el primero, los inherentes al paciente como sus comorbilidades y el segundo, a la complejidad y agresividad del procedimiento quirúrgico. Ponderaron los factores de acuerdo al valor del OR; no determinaron una estratificación, tampoco recomendaciones específicas de acuerdo al tipo de factores presentes, sólo mencionaron que una vez que se identifique un paciente de alto riesgo tiene que recibir tratamiento profiláctico para evitar la trombosis hasta su completa deambulación. En los pacientes de riesgo moderado recomiendan la profilaxis de

\section{Tabla 4: Ponderación y clasificación de los factores de acuerdo a su impacto y determinación del OR} según Anderson. ${ }^{41,44}$

\begin{tabular}{lll}
\hline Riesgo leve OR $<2$ & Riesgo moderado OR 2-9 & Riesgo alto OR $>10$ \\
\hline $\begin{array}{l}\text { Reposo en cama por más de } \\
\text { tres días }\end{array}$ & Cirugía artroscópica de rodilla & Fractura de cadera o miembro inferior \\
$\begin{array}{l}\text { Reposo prolongado } \\
\text { Ancianos }\end{array}$ & $\begin{array}{l}\text { Catéteres venosos centrales } \\
\text { Quimioterapia }\end{array}$ & $\begin{array}{l}\text { Reemplazo de cadera o rodilla } \\
\text { Cirugía mayor }\end{array}$ \\
Obesidad & Falla cardiaca o respiratoria & Trauma mayor \\
& crónica & \\
Venas varicosas & Tumores malignos & Lesión de la médula espinal \\
Embarazo/ante parto & Terapia anticonceptiva oral & \\
& EVC paralítico & \\
& Embarazo/puerperio & \\
& Embolismo previo & \\
& Trombofilia & \\
\hline
\end{tabular}


siete a 10 días; en los pacientes de alto riesgo los cuidados se prolongan hasta cuatro semanas (Tabla 4).

\section{Consideraciones a la clasificación de riesgo de Anderson}

- Los autores consideran que no todos los factores tienen el mismo valor en los estudios clínicos retrospectivos y prospectivos; ponderan los factores de acuerdo al OR. Es una estrategia útil para elaborar una escala con mayor fundamento metodológico.

- Al igual que otras clasificaciones, no están incluidos los factores trombogénicos relacionados a los procedimientos de cirugía plástica.

Consideraciones que nos ayudarán a elegir los mejores cuidados profilácticos para prevenir la trombosis en nuestros pacientes

Deambulación temprana y posición adecuada. La posición adecuada del paciente en la mesa de operaciones es una medida lógica que debe aplicarse a todos los pacientes de cirugía, independientemente de su riesgo. Con respecto a la posición, la técnica consiste en colocar al paciente de tal manera que maximice el flujo venoso a través de las piernas y evite la presión externa. El flujo máximo de sangre a través de la vena poplítea se produce con la rodilla ligeramente flexionada de 10 a 15 grados. Colocar una almohada debajo de las rodillas ayuda a lograr esta función. Se recomienda la ambulación temprana para todos los riesgos, independientemente de los otros cuidados terapéuticos. En el grupo de bajo riesgo estos cuidados son suficientes para reducir eficazmente el riesgo de tromboembolismo venoso. En los pacientes de riesgo moderado o alto, al recuperar la deambulación completa se podrá prescindir de los anticoagulantes. ${ }^{38-42,44}$

Aspirina y antagonistas de la vitamina $\mathrm{K}$. Varios estudios han demostrado menor eficacia de la aspirina en la prevención de la trombosis. También se ha encontrado mayor sangrado del tubo digestivo y de las heridas, este efecto es mayor cuando se combina con anticoagulantes orales como clopidogrel, por eso es un medicamento poco útil en la profilaxis antitrombótica.
El uso de antagonistas de la vitamina $\mathrm{K}$ como el rivaroxabán o apixabán no ha demostrado su eficacia en la prevención de la trombosis y los ha relacionado a hemorragias en pacientes quirúrgicos. ${ }^{38-42,44}$

Medias elásticas de compresión. Las medias ejercen compresión constante en la pantorrilla, evita la estasis venosa y facilita el retorno venoso. Son de mucha utilidad combinadas con heparina de bajo peso molecular o con compresión neumática intermitente. Hacen falta estudios para demostrar su eficacia al usarlas solas o en combinación con otros cuidados. ${ }^{38-42,44,50}$

Compresión neumática intermitente. Actúa por dos mecanismos: uno, reduciendo el estancamiento sanguíneo y facilitando el retorno por el sistema venoso profundo y el otro, por un incremento de la actividad fibrinolítica. Ha sido utilizado en combinación con heparina de bajo peso molecular en la prevención de trombosis en pacientes de riesgo alto y muy alto. Es recomendable en pacientes operados con anestesia general y se puede combinar con medias elásticas. El uso deberá continuar hasta la deambulación del paciente. ${ }^{38-42,44,50}$ En caso de trabajar miembros pélvicos se recomienda colocarlos en brazos para estimular la acción fibrinolítica. Se recomienda iniciar la compresión 30 minutos antes de la inducción.

Heparina fraccionada $y$ heparina de bajo peso molecular. Las dos son recomendadas por ACCP en los pacientes de riesgo moderado a riesgo muy alto. La heparina de bajo peso molecular está disponible, requiere de mínimos cuidados, es más cara, pero requiere de menos aplicaciones; además, en la dosis adecuada ocasiona menor sangrado. Se recomienda aplicarla de ocho a 12 horas después de la cirugía y continuar hasta que el paciente esté completamente deambulando. No hay evidencia de que la aplicación durante la cirugía reduzca el riesgo de trombosis. ${ }^{38-42,44,51-55}$

Warfarina. Está indicada en los pacientes de muy alto riesgo, en estos pacientes la alternativa es el uso de heparina de bajo peso molecular. Este medicamento se debe utilizar en forma combinada con la presión neumática intermitente, medias elásticas y deambulación temprana. Interactúa con diferentes sustancias o medicamentos y requiere de un control estricto de laboratorio. ${ }^{39}$ 
Rivaroxaban. Parece tener mayor eficacia, pero hay riesgo más alto de sangrado en comparación con las heparinas de bajo peso molecular. ${ }^{59}$

Nuevos medicamentos. Han aparecido nuevos medicamentos con acción más específica sobre el factor Xa como hirudina recombinada, fondaparinux, dalteparina y otros más. Algunos de ellos son muy prometedores, pero no hay estudios suficientes para dar una recomendación basada en evidencia. ${ }^{35-39}$

Combinación de cuidados preventivos. El uso combinado de los medios mecánicos y quimioprofilaxis ha demostrado ser el mejor medio en la prevención de la trombosis. ${ }^{35-39,46}$

\section{Calificación del riesgo de trombosis y recomendaciones profilácticas del caso}

Presentamos las calificaciones obtenidas con las diferentes clasificaciones. La calificación con la escala de Caprini-Pannucci fue de un riesgo moderado, la calificación de Davison y la de IMPROVE fue considerada como riesgo muy alto. Existe una marcada diferencia en la clasificación del riesgo; sin embargo, hay una concordancia en la recomendación del uso de heparina de bajo peso molecular y de cuidados generales. La paciente presenta algunos factores trombogénicos que las clasificaciones actuales no incluyen como viaje en avión en clase turista, edema de extremidades, liposucción e infiltración grasa en los glúteos y varios procedimientos en una sola cirugía. Si estos factores fueran incluidos, la calificación del riesgo sería mayor y obligaría a tener mayores cuidados profilácticos y prolongar su aplicación hasta seis semanas después de la cirugía. En las pacientes que tienen que realizar un viaje en avión para acudir a su cirugía es importante mantener una vigilancia estrecha perioperatoria en la detección oportuna de trombosis. Se recomienda el uso del ultrasonido Doppler para este fin (Tabla 5).,35,56-66

En esta paciente es altamente recomendable:

- Prepararla varios meses antes de la cirugía.

- Disminuir el peso corporal.

- Suspender los estrógenos de cuatro a seis semanas antes de la cirugía.

- Viajar como mínimo una semana antes y de tres a cuatro semanas después de la cirugía. Sugerir que viaje en clase turista evitando la deshidratación, los alimentos que aumenten la inflamación y las bebidas alcohólicas. Recomendar hacer ejercicios durante el viaje.

- En días previos a la cirugía se requiere una valoración integral que busque signos de trombosis. Es recomendable realizar una buena exploración física, exámenes de laboratorio y un estudio con ultrasonido Doppler perioperatorio.

- Disminuir el número de procedimientos y acortar los tiempos quirúrgicos.

- Aplicar heparina de bajo peso molecular 12 horas después de la cirugía y cuidados gene-

\begin{tabular}{|c|c|c|c|c|c|c|}
\hline $\begin{array}{l}\text { Clasifica- } \\
\text { ción }\end{array}$ & & & & & Total & Riesgo \\
\hline $\begin{array}{l}\text { Caprini- } \\
\text { Pannucci } \\
\text { ACCP } \\
\text { (CHEST) }\end{array}$ & $\begin{array}{l}\text { Edad de } 48 \text { años } \\
\text { = } 1 \text { punto } \\
\text { Mayor de } 40 \\
\text { años }\end{array}$ & $\begin{array}{c}\text { IMC } 33.6= \\
1 \text { punto } \\
\text { Varios factores }\end{array}$ & $\begin{array}{c}\text { Hormonas }= \\
1 \text { punto }\end{array}$ & $\begin{array}{c}\text { Cirugía mayor = } \\
2 \text { puntos } \\
\text { Cirugía mayor }\end{array}$ & 5 puntos & $\begin{array}{c}\text { Moderado } \\
\text { Alto }\end{array}$ \\
\hline Davison & $\begin{array}{l}\text { Edad de } 40 \text { a } 60 \\
\text { años }=1 \text { factor }\end{array}$ & $\begin{array}{c}\text { Obesidad = } \\
1 \text { factor }\end{array}$ & $\begin{array}{c}\text { Hormonas }= \\
1 \text { factor }\end{array}$ & $\begin{array}{c}\text { Cirugía mayor = } \\
2 \text { factores }\end{array}$ & 5 factores & Muy alto \\
\hline IMPROVE & $\begin{array}{c}\text { Mayor de } 40 \\
\text { años }\end{array}$ & Varios factores & & $\begin{array}{l}\text { Varios pro- } \\
\text { cedimientos } \\
\text { importantes }\end{array}$ & & Muy alto \\
\hline
\end{tabular}


rales como posición adecuada, ángulo de la rodilla de 10 a 15 grados, pies ligeramente elevados, deambulación temprana, compresión neumática intermitente, medias elásticas, prolongar la profilaxis de cuatro a seis semanas después de la cirugía hasta varias semanas después de que la paciente haya retornado a su domicilio.

\section{DISCUSIÓN Y CONCLUSIONES}

La clasificación del riesgo de tromboembolia y la aplicación de cuidados preventivos han disminuido considerablemente la incidencia de trombosis, incluyendo la TEP mortal. Todos los autores coinciden en la importancia de clasificar a los pacientes de acuerdo al riesgo de trombosis y en la aplicación de profilaxis; sin embargo, no hay una uniformidad en la clasificación, las escalas actuales no incluyen factores trombogénicos existentes en los pacientes de cirugía plástica. Todos los pacientes que se someten a una cirugía tienen el riesgo de complicarse con trombosis, incluso los pacientes a los que se les realiza cirugía facial (lifting facial), por eso todos deberán tener una valoración preoperatoria y una calificación de riesgo de trombosis. ${ }^{15,18,21,26,57,67-73}$ Mientras no haya una escala efectiva adaptada a los pacientes de cirugía plástica, se deberá utilizar una clasificación existente y se deberán tener en cuenta los factores trombogénicos de los procedimientos de la especialidad, lo que aumentará la calificación de los pacientes y la indicación de la profilaxis. Los cuidados preventivos son importantes para evitar esta complicación, los mejores resultados se obtienen con el uso combinado de la quimioprofilaxis y la aplicación de cuidados generales. Cuando se requiera el uso de quimioprofilaxis, hay que utilizar heparinas de bajo peso molecular o heparina no fraccionada. No existen estudios suficientes en el uso de anticoagulantes orales que demuestren su eficacia y seguridad. El comité de seguridad está realizando estudios clínicos para identificar los factores de riesgo de la trombosis y elaborar una escala con una mayor sensibilidad y especificidad adaptada a los pacientes de la especialidad para determinar la eficacia de la profilaxis. Todavía requerimos de tiempo para su culminación.

\section{REFERENCIAS}

1. Cabrera-Rayo A, Nellen-Hummel H. Epidemiología de la enfermedad tromboembólica venosa. Gac Med Mex 2007; 143 (Supl 1): 3-5.

2. Zaragoza-Delgadillo JC, Sauri-Arce JCA, Ochoa-Carrillo FJ, Fuentes-del-Toro F. Prevención de la enfermedad tromboembólica venosa en cirugía. Gac Med Mex 2007; 143 (Supl 1): 35-39.

3. Saad AN, Parina R, Chang D, Gosman AA. Risk of adverse outcomes when plastic surgery procedures are combined. Plast Reconstr Surg 2014; 134 (6): 1415-1422.

4. Rao RB, Ely SF, Hoffman RS. Deaths related to liposuction. N Eng J Med 1999; 340 (19): 1471-1475.

5. Ramírez-Guerrero JA. Liposucción. Consideraciones anestésicas y perioperatorias. Rev Mex Anest 2007; 30 (4): 233-241.

6. Grazer FM, Jong RH. Fatal outcomes from liposuction. Census survey of cosmetic surgeons. Plast Reconstr Surg 2000; 105: 436-446.

7. Feisher LA. Risk of anesthesia. In: Miller RD, Ed. Anesthesia. Churchill Livingstone; 2005. pp. 893-920.

8. Katz BE, Bruck MC, Felsenfeld $L$ et al. Power liposuction: report on complications. Dermatol Surg 2003; 29: 925-927.

9. Hanke CW, Bullock S, Bernstein G. Current status of tumescent liposuction in the United States: national survey results. Dermatol Surg 1996; 22: 595-598.

10. Hoefflin SM, Bornstein JB, Gordon M. General anesthesia in an office-based plastic surgical facility: a report on more than 23000 consecutive officebased procedures under general anesthesia with no significant anesthetic complications. Plats Reconstr Surg 2001; 107: 243-251.

11. Scarborough DA, Herron JB, Khan A, Bisaccia E. Experience with more than 5,000 cases in which monitored anesthesia care was used for liposuction surgery. Aesth Plast Surg 2003; 27: 474-480.

12. Hanke CW, Bernstein G, Bullock S. Safety of tumescent liposuction in 15,336 patients. National survey results. Dermatol Surg 1995; 21: 459-462.

13. Johnson PJ. General anesthesia in an office-based plastic surgical facility: a report on more than 23,000 consecutive office-based procedures under general anesthesia with no significant anesthetic complications. Arch Facial Plast Surg 2001; 3: 287.

14. Ibarra P, Arango J, Bayter J, Castro J, Cortes J et al. Consenso de la Sociedad Colombiana de Anestesiología y Reanimación, SCARE y de la Sociedad Colombiana de Cirugía Plástica, sobre las recomendaciones para el manejo de pacientes electivos de bajo riesgo. Rev Col Anest 2010; 37 (4): 390-403.

15. Winocour J, Gupta V, Ramírez JR, Shack RB, Grotting JC, Higdon KK. Abdominoplasty: risk factor, complications rates, and safety of combined procedures. Plast Reconstr Surg 2015; 136: 597e-606e.

16. Poore SO, Sillah N, Mahajan AY, Gotowski KA. Patient safety in the operating room: II intraoperative and postoperative. Plast Reconstr Surg 2012; 130: 10481058.

17. Cárdenas-Salomón CM, Gutiérrez-Salgado JE, Márquez-Espriella C, Sánchez-Medal FP, GallardoPalacios GJ, Eljure-Eljure M. Tromboprofilaxis en 
Cirugía Plástica: ¿Cuándo, cómo y por qué? Cir Plast 2013; 23 (3): 168-178.

18. Sánchez HA. Profilaxis de la enfermedad tromboembólica venosa. Cuad Cir 2000; 14: 44-54.

19. Lenhart M, Homann HH, Daigeler A et al. Major and lethal complication of liposuction. Plast Reconstr Surg 2008; 121: 396e-403e.

20. Pérez-García A, Briones-Pérez. Tromboprofilaxis en pacientes postquirúrgicos: revisión de 1,500 casos. Cir Cir 2004; 72: 287-291.

21. Pannucci ChJ. Evidence-based Recipes for venous thromboembolism prophylaxis: a practical safety guide. Plast Reconstr Surg 2017; 139: 520e-532e.

22. Caprini JA, Arcelus JI, Reyna JJ. Effective risk stratification of surgical and nonsurgical patients for venous thromboembolic disease. Semin Hematol 2001; 38: 12-19.

23. Pannucci CJ, Bailey SH, Dreszer G, Fisher-Wachtman C, Zumsteg JW, Jaber RM et al. Validation of the Caprini risk assessment model in plastic and reconstructive surgery patients. J Am Coll Surg 2011; 212 (1): 105112.

24. Murphy RX Jr, Alderman A, Gutowski K, Kerrigan C, Rosolowski K, Schechter L et al. Evidence-based practices for thromboembolism prevention: summary of the ASPS Venous Thromboembolism Task Force Report. Plast Reconstr Surg 2012; 130 (1): 168e-175e.

25. Cronin MA, Demgler N, Krauss ES, Segal A, Wei N, Daly $\mathrm{M}$ et al. Completion of the update Caprini risk assessment model (2013 version). Clin Appl Thromb Haemost 2019; 25: 1-10.

26. Swanson E. The case against chemoprophylaxis for venous thromboembolism prevention and the rationale for SAFE anesthesia. Plast Reconstr Surg Glob Open 2014; 2: e160.

27. Cuenca-Pardo J, Ramos-Gallardo G, CárdenasCamarena L, Contreras-Bulnes L, Lelevier-de Avelar G. Searching for the best way to assess the risk of thrombosis in aesthetic plastic surgery; the role of the Caprini/Pannucci score. Aesth Plast Surg 2019; 43 (5): 1387-1395.

28. Turpie AG, Bauer KA, Caprini JA et al; Apollo Investigators. Fondaparinux combined with intermittent pneumatic compression vs. Intermittent pneumatic compression alone for prevention of venous thromboembolism after abdominal surgery: a randomized, double-blind comparison. J Thromb Haemost 2007; 5 (9): 1854-1861.

29. Kim EK, Eom JS, Ahn SH et al. The efficacy of prophylactic low-molecular-weight heparin to prevent pulmonary thromboembolism in immediate breast reconstruction using the TRAM flap. Plast Reconstr Surg 2009; 123 (1): 9-12.

30. Edwards JZ, Pulido PA, Ezzet KA et al. Portable compression device and low-molecular-weight heparin compared with low-molecular-weight heparin for thromboprophylaxis after total joint arthroplasty. J Arthroplasty 2008; 23 (8): 1122-1127. Epub 2008 Apr 2.

31. Senaran $\mathrm{H}$, Acaroglu E, Ozdemir HM et al. Enoxaparin and heparin comparison of deep vein thrombosis prophylaxis in total hip replacement patients. Arch Orthop Trauma Surg 2006; 126 (1): 1-5.
32. Chin PL, Amin MS, Yang KY et al. Thromboembolic prophylaxis for total knee arthroplasty in Asian patients: a randomized controlled trial. J Orthop Surg (Hong Kong) 2009; 17 (1): 1-5.

33. Colwell CW Jr, Kwong LM, Turpie AG et al. Flexibility in administration of fondaparinux for prevention of symptomatic venous thromboembolism in orthopedic surgery. J Arthroplasty 2006; 21 (1): 36-45.

34. Barrellier MT, Level B, Parienti JJ et al. Short versus extended thromboprophylaxis after total knee arthroplasty: a randomized comparison. Thromb Res 2010; 126 (4): e298-e304.

35. Bottaro FJ, Elizondo MC, Doti C, Bruetman JE et al. Efficacy of extended thromboprophylaxis in major abdominal surgery: what does the evidence show? A meta-analysis. J Thromb Haemost 2008; 99 (6): 1104-1111.

36. Rasmussen MS, Jorgensen LN, Wille-Jørgensen $P$ et al; FAME Investigators. Prolonged prophylaxis with dalteparin to prevent late thromboembolic complications in patients undergoing major abdominal surgery: a multicenter randomized open-label study. J Thromb Haemost 2006; 4 (11): 2384-2390.

37. Rasmussen MS, Jørgensen LN, Wille-Jørgensen P. Prolonged thromboprophylaxis with low molecular weight heparin for abdominal or pelvic surgery. Cochrane Database Syst Rev 2009; 21 (1): CD004318.

38. American College of Chest Physicians Antithrombotic Guidelines, 9th ed. Accessed November 13, 2013. Available at: http: //www.chestnet.org/Guidelines-andResources/Guidelines-and-Consensus- Statements/ Antithrombotic-Guidelines-9th-Ed.

39. Geerts WH, Bergqvist D, Pineo GF, Heit JA, Samama CM, Lassen MR et al. Prevention of venous thromboembolism: American College of Chest Physicians Evidence-Based Clinical Practice Guidelines (8th Edition). Chest 2008; 133 (6 Suppl): 381S-453S.

40. Guyatt GH, Akl EA, Crowther M, Gutterman DD, Schuünemann HJ; American College of Chest Physicians Antithrombotic Therapy and Prevention of Thrombosis Panel. Executive summary: Antithrombotic Therapy and Prevention of Thrombosis, 9th ed: American College of Chest Physicians Evidence-Based Clinical Practice Guidelines. Chest 2012; 141 (2 Suppl): 7S-47S.

41. Morales-Blanhir JE, Salas-Pacheco JL, Rosas-Romero MJ, Valle-Murillo MA. Diagnóstico de tromboembolia pulmonar. Arch Cardiol Mex 2011; 81 (2): 126-136.

42. Davison SP, Venturi ML, Attinger Ch E, Baker SB, Spear SL. Prevention of venous thromboembolism in the plastic surgery patient. Plast Reconstr Surg 2004; 114 (3): 43e-51e.

43. Guyatt G, Gutterman D, Baumann MH, AddrizzoHarris D, Hylek EM, Phillips B et al. Grading strength of recommendations and quality of evidence in clinical guidelines: report from an American College of Chest Physicians Task Force. Chest 2006; 129 (1): 174-181.

44. Anderson FA, Spencer FA. Risk factors for venous thrombolism. Circulation 2003; 107: 19-16.

45. Henke P, Pannucci $\mathrm{CH}$. VTE risk factor assessment and prophylaxis. Phlebology 2010; 25 (5): 219-223.

46. Bumaschny E, Rafa CA, Reichman P. Evaluación preoperatoria del paciente quirúrgico. En: Galindo F, et al. Enciclopedia de cirugía digestiva. www.sacd.org. ar 2013; tomo I-101, pp. 1-20. 
47. Pastor-Torres LF, Antigao-Ramírez R, Honorato-Pérez $J M$, Junquera-Planas CM, Navarro-Salas E et al. Guías de práctica clínica de la Sociedad Española de Cardiología en la valoración del riesgo quirúrgico del paciente cardiópata sometido a cirugía no cardiaca. Rev Esp Cardiol 2001; 54: 186-193.

48. www.outcomes-umassmed.org/IMPROVE/risk_score/ index.html / www.DVT.ORG

49. www.fundaciontorax.org/calc/embop/ScoreTVP.html

50. Kakkos SK, Caprini JA, Geroulakos G, Nicolaides AN, Stansby GP, Reddy DJ. Combined intermittent pneumatic leg compression and pharmacological prophylaxis for prevention of venous thromboembolism in high-risk patients. Cochrane Database Syst Rev 2008; (4): CD005258.

51. Dini GM, Ferreira MC, Albuquerque LG et al. How safe is thromboprophylaxis in abdominoplasty? Plast Reconstr Surg 2012; 130: 851e-857e.

52. Pannucci CJ, Dreszer G, Wachtman CF et al. Postoperative enoxaparin prevents symptomatic venous thromboembolism in high-risk plastic surgery patients. Plast Reconstr Surg 2011; 128: 1093-1103.

53. Enoxaparin Side Effects. Accessed November 13, 2013. Available at: http: //www.drugs.com/sfx/enoxaparinside-effects.html.

54. Hatef DA, Kenkel JM, Nguyen MQ et al. Thromboembolic risk assessment and the efficacy of enoxaparin prophylaxis in excisional body contouring surgery. Plast Reconstr Surg 2008; 122: 269-279.

55. Pannucci CJ, Wachtman CF, Dreszer G et al. The effect of post-operative enoxaparin on risk for re-operative hematoma. Plast Reconstr Surg 2012; 129: 160-168.

56. Gómez-Outes A, Terleira-Fernández AI, Suárez-Gea ML, Vargas-Castrillón E. Dabigatran, rivaroxaban, or apixaban versus enoxaparin for thromboprophylaxis after total hip or knee replacement: Systematic review, meta-analysis, and indirect treatment comparisons. BMJ 2012; 344: e3675.

57. Homans J. Thrombosis of the deep leg vein due to prolonged sitting. N Engl J Med 1954; 250: 148149.

58. Adabi K, Stern CS, Weichman KE, Garfein ES, Pothula A, Draper L et al. Population health implications of medical tourism. Plast Reconstr Surg 2017; 140 (1): 66-74.

59. Franzblau LE, Chung KC. Impact of medical tourism on cosmetic surgery in the United States. Plast Reconstr Surg Glob Open 2013; 1 (7): e63.

60. Ross KM, Moscoso AV, Bayer LR, Rosselli-Risal CL, Orgill DP. Plastic surgery complications from medical tourism treated in a U.S. Academic Medical Center. Plast Reconstr Surg 2018; 141 (4): 517-523.

61. Ríos-Tejada F, Villegas-Fernández F, Azofra-García JA, Callol-Sánchez L. Síndrome del pasajero de clase económica. An Med Int (Madrid) 2002; 19 (11): 589593.

62. Ten Wolde M, Quak E, Prins MH, Kraaijenhagen RA, Buller $\mathrm{HR}$. Long distance travelling increase risk of pulmonary embolism. Thromb Haemost 2001; 86 (Suppl).

63. Cheung B, Duflou J. Pre-existing pulmonary thromboembolic disease in passengers with the «economy class syndrome». Aviat Space Environ Med 2001; 72: 747-749.

64. Sanabria-Candla FA, Vaga-Cruz PJ. Tromboembolismo pulmonar: síndrome del viajero o síndrome de la clase turista. Rev Científica Médica 2005; 8 (1): 35-37.

65. Suárez C. Síndrome de la clase turista. Med Clin (Barc) 2002; 119 (1): 16-17.

66. Belcaro G, Geroulakos G, Nicolaides AN, Myers KA, Winfoed $M$. Venous thromboembolism from air travel: the LONFLIT study. Angiology 2001; 52: 369-374.

67. Machin SJ, Mackie IJ, McDonald S, Bailey-King S, Colleridge-Smith P, Scurr JH. Airline travel: incidence and prevention of venous thrombosis. Thromb Haemost 2001; 87 (Suppl): 369-374.

68. Swanson E. Prospective clinical study of 551 cases of liposuction and abdominoplasty performed individually and in combination. Plast Reconstr Surg Glob Open 2013; 1: e32.

69. Reinish JF, Bresnick SD, Walker JW, Rosso RF. Deep venous thrombosis and pulmonary embolus after face lift: A study of incidence and prophylaxis. Plast Reconstr Surg 2001; 107: 1570.

70. Stuzin JM, Baker TJ, Baker TM. Deep venous thrombosis and pulmonary embolus after face lift: a study of incidence and prophylaxis (discussion). Plast Reconstr Surg 2001; 107: 1576-1577.

71. Durnig P, Jungwirth W. Low-molecular-weight heparin and postoperative bleeding in rhytidectomy. Plast Reconstr Surg 2006; 118: 502-507; discussion 508.

72. Seruya $M$, Venturi $M L$, lorio $M L$ et al. Efficacy and safety of venous thromboembolism prophylaxis in highest risk plastic surgery patients. Plast Reconstr Surg 2008; 122: 1701-1708.

73. Pannucci CJ, MacDonald JK, Ariyan S et al. Benefits and risks of prophylaxis for deep venous thrombosis and pulmonary embolus in plastic surgery: A systematic review and meta-analysis of controlled trials and consensus conference. Plast Reconstr Surg 2016; 137: 709-730.
Correspondencia:
Dr. Jesús Cuenca Pardo
Jerez Núm. 51, Col. Mixcoac,
Alcaldía Benito Juárez, 03220,
Ciudad de México, México.
E-mail: jcuenca001@gmail.com 


\title{
RECOMENDACIONES DE SEGURIDAD
}

\section{Estratificación de riesgo de trombosis}

\section{y profilaxis Escala de Caprini}

\author{
Trombosis \\ Trombosis venosa profunda \\ Tromboembolia pulmonar
}
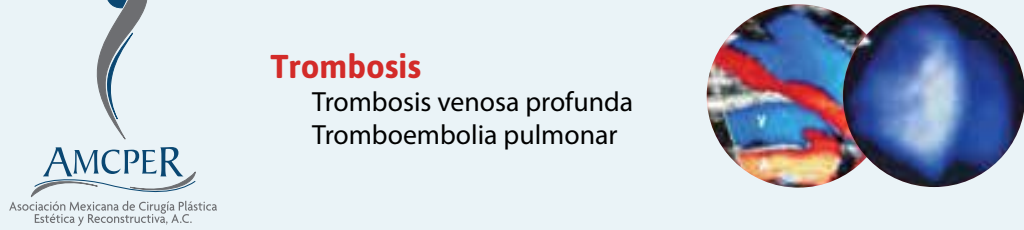

La trombosis es una complicación frecuente en los procedimientos de cirugía plástica; es la principal complicación mortal en pacientes que se les realiza abdominoplastia; la incidencia aumenta, cuando se combina con liposucción o algún otro tipo de procedimiento.

\begin{tabular}{|c|c|c|c|}
\hline 1 punto & 2 puntos & 3 puntos & 5 puntos \\
\hline Edad de 41-60 años & Edad 61-74 años & Edad mayor 75 años & $\begin{array}{c}\text { Fractura de cadera o } \\
\text { miembro pélvico }\end{array}$ \\
\hline Cirugía menor $<45$ minutos & Cirugía mayor (45 minutos) & Antecedentes de TVP o TEP & $\begin{array}{l}\text { Reemplazo de cadera, } \\
\text { pelvis o rodilla }\end{array}$ \\
\hline Cirugía mayor último mes & Malignidad presente o previa & Historia familiar trombosis & Evento cerebral vascular \\
\hline Venas varicosas visibles & Cirugía artroscópica & Factor V de Leiden & Mieloma múltiple \\
\hline $\begin{array}{c}\text { Enfermedad inflamatoria } \\
\text { intestinal }\end{array}$ & $\begin{array}{l}\text { Cirugía laparoscópica } \\
\text { (45 minutos) }\end{array}$ & $\begin{array}{c}\text { Presencia de } \\
\text { protrombina 20210A }\end{array}$ & $\begin{array}{l}\text { Lesión de la médula } \\
\text { espinal (parálisis) }\end{array}$ \\
\hline Edema de piernas & & Homocisteína elevada & Derrame cerebral \\
\hline IMC mayor 25 & $\begin{array}{l}\text { Yeso inmovilizador } \\
\text { último mes }\end{array}$ & Anticoagulante de lupus & \\
\hline Ataque al corazón & $\begin{array}{l}\text { Presencia acceso } \\
\text { venoso central }\end{array}$ & Anticuerpos anticardiolipina & $\begin{array}{c}\text { SÓLO PARA MUJERES } \\
1 \text { PUNTO }\end{array}$ \\
\hline Falla cardiaca congestiva & & $\begin{array}{l}\text { Trombocitopenia } \\
\text { inducida por heparina }\end{array}$ & $\begin{array}{l}\text { Anticonceptivos o terapia } \\
\text { hormonal de reemplazo }\end{array}$ \\
\hline Severa infección último mes & & $\begin{array}{c}\text { Otra trombofilia } \\
\text { congénita o adquirida }\end{array}$ & $\begin{array}{c}\text { Embarazo o parto } \\
\text { último mes }\end{array}$ \\
\hline Enfermedad pulmonar & & & $\begin{array}{l}\text { Abortos espontáneos } \\
\text { recurrentes }\end{array}$ \\
\hline Reposo actual en cama & $\begin{array}{l}\text { Confinamiento en cama } \\
\text { por } 72 \text { horas o más }\end{array}$ & & \\
\hline $\begin{array}{l}\text { Otros factores de riesgo; } \\
1 \text { punto por cada uno }\end{array}$ & & & \\
\hline
\end{tabular}

\section{Escala de Caprini/Pannucci Faltan factores propios de las pacientes de cirugía plástica}

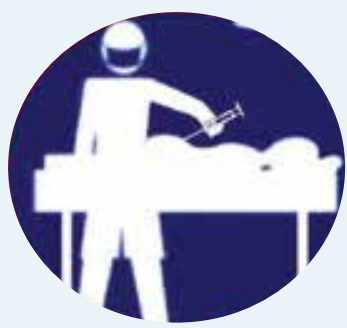

- Abdominoplastia sola o combinada con cesárea

- Cirugía de múltiples procedimientos

- Liposucción e infiltración de grasa

- Viaje reciente en avión

- Ingesta de hormonales

- Obesidad

- Colagenopatías

Mujeres menores de 35 años con más de 2 de estos factores, tienen un alto riesgo de trombosis, deben calificarse con una puntuación alta
Cuenca-Pardo J, Ramos-Gallardo G, CárdenasCamarena L, Contreras-Bulnes L, Lelevier De Alvear G. Searching for the Best Way to Assess the Risk of Thrombosis in Aesthetic Plastic Surgery: The Role of the Caprini/Pannucci Score. Aesthetic Plast Surg 2019; https://doi.org/10.1007/s00266-019-01428-z

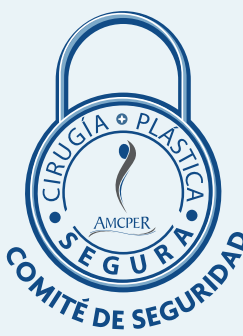

Son muchas las escalas para calificar el riesgo de trombosis

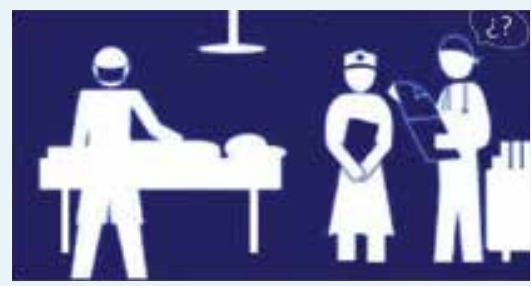

Las más utilizadas en Cirugía Plástica son: Caprini/Pannucci ACCP (CHEST)

\section{Escalas para estratificar} el riesgo de trombosis

- IMPROVE

- PADUA

- DAVISON

- CAPRINI/PANNUCCI

- ACCP (CHEST)

- BAYTER

- NORTHSHORE UNIVERSITY HEALTH

- ANDERSON

\begin{tabular}{|c|c|c|}
\hline $\begin{array}{l}\text { A. Factores que deben } \\
\text { ser incluidos en la escala } \\
\text { según Caprini; dar } 1 \\
\text { punto extra por cada uno }\end{array}$ & $\begin{array}{l}\text { B. Factores } \\
\text { trombogénicos presentes } \\
\text { en las pacientes } \\
\text { de procedimientos } \\
\text { estéticos; aún no se han } \\
\text { ponderado, se debe dar } \\
\text { por lo menos } 1 \text { punto } \\
\text { cada factor }\end{array}$ & $\begin{array}{l}\text { C. Pacientes menores } \\
\text { a } 35 \text { años con los } \\
\text { siguientes antecedentes, } \\
\text { se debe dar una mayor } \\
\text { calificación; aún no están } \\
\text { ponderadas, pero debe } \\
\text { ser mayor a un punto }\end{array}$ \\
\hline $\begin{array}{l}\text { Obesidad mórbida } \\
\text { IMC }>40\end{array}$ & $\begin{array}{c}\text { Viajes en avión o } \\
\text { autobús previos } \\
\text { a la cirugía }\end{array}$ & $\begin{array}{l}\text { Infiltración de grasa } \\
\text { en los glúteos }\end{array}$ \\
\hline Tabaquismo & Abdominoplastia & Venas varicosas \\
\hline $\begin{array}{l}\text { Diabéticos que } \\
\text { requieren insulina }\end{array}$ & $\begin{array}{l}\text { Plastia de la pared } \\
\text { abdominal }\end{array}$ & Obesidad \\
\hline Quimioterapia & Liposucción & Colagenopatías \\
\hline $\begin{array}{l}\text { Transfusiones } \\
\text { sanguineas }\end{array}$ & Infiltración de grasa & $\begin{array}{l}\text { Abdominoplastia } \\
\text { en el mismo tiempo } \\
\text { quirúrgico de una } \\
\text { cesárea o parto vaginal }\end{array}$ \\
\hline SIDA (VIH) & $\begin{array}{l}\text { Cirugías combinadas } \\
\text { o múltiples }\end{array}$ & $\begin{array}{l}\text { Procedimientos } \\
\text { múltiples }\end{array}$ \\
\hline \multirow[t]{2}{*}{$\begin{array}{l}\text { Tiempo de cirugía } \\
\text { mayor a } 2 \text { horas }\end{array}$} & $\begin{array}{l}\text { Grado de movilidad } \\
\text { y participación }\end{array}$ & Viajes en avión \\
\hline & & Ingesta de hormonales \\
\hline
\end{tabular}


Estratificación del riesgo de trombosis y profilaxis

\section{Recomendaciones profilácticas; Caprini/Pannucci}

3 puntos. Profilaxis mecánica y química, el tiempo que no camine el paciente.

3-6 puntos. Heparina de bajo peso molecular o heparina no fraccionada, por una semana.

7 o más puntos. Preparar al paciente para disminuir la puntuación. Heparina de bajo peso molecular; mínimo 4 semanas.

1. Pannucci ChJ. Evidence-based Recipes for venous thromboembolism prophylaxis: a practical safety guide. Plast Reconstr Surg 2017; 139: 520e-532e.

2. Caprini JA, Arcelus JI, Reyna JJ. Effective risk stratification of surgical and nonsurgical patients for venous thromboembolic disease. Semin Hematol 2001; 38: 12-19.

3. Pannucci CJ, Bailey SH, Dreszer G, Fisher-Wachtman C, Zumsteg JW, Jaber RM et al. Validation of the Caprini risk assessment model in plastic and reconstructive surgery patients. J Am Coll Surg 2011; 212 (1): 105-112.

4. Murphy RX Jr, Alderman A, Gutowski K, Kerrigan C, Rosolowski K, Schechter L et al. Evidence-based practices for thromboembolism prevention: summary of the ASPS Venous Thromboembolism Task Force Report. Plast Reconstr Surg 2012; 130 (1): 168e-175e.

5. Cronin MA, Demgler N, Krauss ES, Segal A, Wei N, Daly M et al. Completion of the update Caprini risk assessment model (2013 version). Clin Appl Thromb Haemost 2019; 25: 1-10.

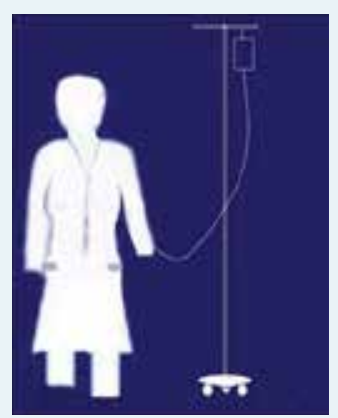

\section{Deambulación temprana en el postoperatorio inmediato}

1. Mejora el flujo venoso

2. Evita el estancamiento sanguíneo y la trombosis
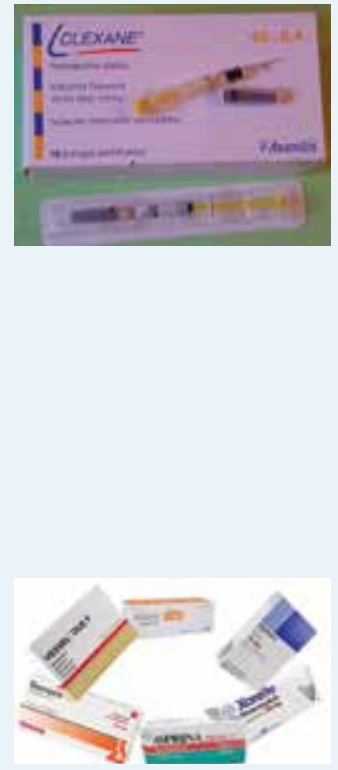

Anticoagulantes y antiagregantes por vía oral

1. Podrán ser de utilidad una vez que se demuestre su eficacia y seguridad en los pacientes de cirugía plástica

2. Podrían ser utilizados en pacientes que requieren una quimio-profilaxis prolongada

3. Combinados en aspirina, aumentan el riesgo de sangrado

\section{Consideraciones que nos ayudarán a elegir los cuidados profilácticos para prevenir la trombosis}

\section{Posición}

Durante la cirugía y en el postoperatorio inmediato

1. Maximizar el flujo venoso de las piernas y evitar la compresión externa

2. La flexión de 10 a 15 grados de la rodilla colocando una almohada, logra esta función

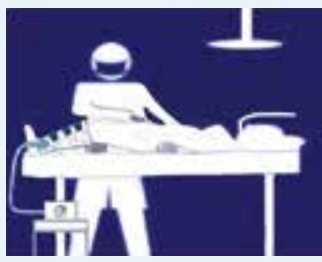

\section{Compresión neumática intermitente Usarlo $\mathbf{3 0}$ minutos previos a la cirugía y hasta que el paciente deambule}

1. Evita el estancamiento y facilita el retorno venoso

2. Estimula la fibrinólisis

3. Puede utilizarse en combinación de medias elásticas

\section{Medias elásticas}

Colocarlas antes de la cirugía y usar hasta que el paciente deambule

1. Mejora la circulación venosa y evita el estancamiento

$\begin{array}{cl}\text { Terapia combinada } & \text { - Heparina bajo peso } \\ \text { ¡Es la mejor profilaxis } & \text { - Ambulación } \\ \text { en la prevención } & \text { - Medias elásticas } \\ \text { de la trombosis! } & \text { - Compresión neumática }\end{array}$

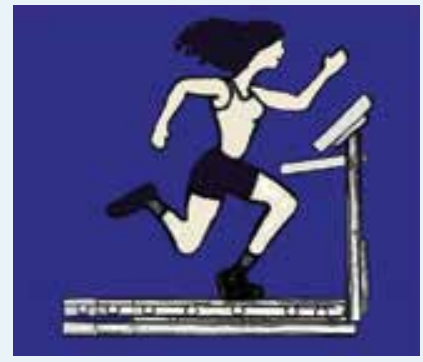

Altamente recomendable para reducir el riesgo:

1. Aumentar el grado de movilidad y participación

2. Controlar las comorbilidades

3. Disminuir el tiempo quirúrgico

4. Evitar los procedimientos múltiples

5. Profilaxis antitrombótica oportuna y adecuada 